\title{
Correction to: Different phytotoxic effect of Lolium multiflorum Lam. leaves against Echinochloa oryzoides (Ard.) Fritsch and Oryza sativa L.
}

\author{
Sara Vitalini ${ }^{1}$ - Francesca Orlando ${ }^{2}$ - Alessandro Palmioli ${ }^{3}$ - Sumer Alali ${ }^{4}$. Cristina Airoldi ${ }^{3} \cdot$ Ivano De Noni $^{5}$. \\ Valentina Vaglia ${ }^{4}$. Stefano Bocchi ${ }^{4}$ - Marcello Iriti ${ }^{1}$ (I)
}

Published online: 17 July 2020

(C) Springer-Verlag GmbH Germany, part of Springer Nature 2020

\section{Correction to: Environmental Science and Pollution Research https://doi.org/10.1007/s11356-020-09573-8}

In the title, it should be Oryza instead of Oriza.

Publisher's note Springer Nature remains neutral with regard to jurisdictional claims in published maps and institutional affiliations.

Francesca Orlando was co-first author and Stefano Bocchi was co-last author.

The online version of the original article can be found at https://doi.org/ 10.1007/s11356-020-09573-8

Sara Vitalini

sara.vitalini@unimi.it

$\triangle$ Marcello Iriti

marcello.iriti@unimi.it

1 Department of Agricultural and Environmental Sciences, Università degli Studi di Milano, Via G. Celoria 2, 20133 Milan, Italy

2 Department of Molecular and Translational Medicine (DMMT), Università degli Studi di Brescia, Viale Europa, 11, 25123 Brescia, BS, Italy
3 Department of Biotechnology and Biosciences, BioOrgNMR Lab, Università degli Studi di Milano Bicocca, Piazza della Scienza 2, 20126 Milan, Italy

4 Department of Environmental Science and Policy, Università degli Studi di Milano, Via G. Celoria 2, 20133 Milan, Italy

Department of Food, Enviromental and Nutritional Sciences, Università degli Studi di Milano, Via G. Celoria 2, 20133 Milan, Italy 